\title{
Improved Hessian multiscale enhancement filter
}

\author{
Jinzhu Yang ${ }^{\mathrm{a}, \mathrm{b}^{*}}$, Shuang Ma ${ }^{\mathrm{a}, \mathrm{b}}$, Qi Sun ${ }^{\mathrm{c}}$, Wenjun Tan ${ }^{\mathrm{a}, \mathrm{b}}$, Mengjia Xu ${ }^{\mathrm{a}, \mathrm{b}}$, Nan Chen ${ }^{\mathrm{d}}$ and \\ Dazhe Zhao ${ }^{\mathrm{a}, \mathrm{b}}$ \\ ${ }^{a}$ Key Laboratory of Medical Image Computing of Northeastern University, Ministry of Education, \\ Shenyang 110819, China \\ ${ }^{b}$ College of Information Science and Engineering, Northeastern University, Shenyang 110004, China \\ ${ }^{c}$ Neusoft, Shenyang 110819, China \\ ${ }^{d}$ Department of Radiology, Xuanwu Hospital, Capital Medical University, Beijing, China
}

\begin{abstract}
Traditional Hessian multiscale filter consider only the local geometric feature but not the global grayscale information. In medical image analysis, Hessian filter is usually used to enhance the blood vessels. However, it also produces some pseudo vascular structures or some isolate noise points, such as the nasal soft tissues that have the similar shape with the vessels in MRA data, which will increase the difficulty of cerebrovascular segmentation. To resolve this issue, an improved Hessian multiscale filter is proposed in this paper. An image grayscale factor is added to the vascular similarity function computed by Hessian matrix eigenvalue. This method is experimented on brain MRA data and lung CTA data. Experimental results show that this method can enhance vascular structures, and simultaneously reduce the appearance of the pseudo vascular structures and the isolated noise points.
\end{abstract}

Keywords: Hessian filter, multiscale enhancement, grayscale, CT image, MRA image

\section{Introduction}

Cardiovascular disease (including heart disease and cerebrovascular disease) has become one of the main hazards to human health. On August 9, 2012, the National Center for Cardiovascular Diseases released the Report on Cardiovascular Diseases in China (2012). According to the report, the number of people with cardiovascular disease has been reached 290 million and nearly 3.5 million people die from cardiovascular disease every year, which amounts to $41 \%$ of the total causes of death, which is highest among all causes of diseases. Cardiovascular diseases kill about 9590 people every day in China, which means that nearly 400 people die from cardiovascular diseases per hour, and one person dies from cardiovascular disease every 10 seconds [1]. Hence the challenge before the medical practitioners is how to diagnose and detect the cardiovascular disease at an early hour. Recently, one of the effective techniques to diagnose and detect this kind of disease is by analyzing the imaging through $\mathrm{CAD}$ technology. There are four main imaging technologies for the clinical diagnosis of cerebrovascu-

${ }^{*}$ Corresponding author: Jinzhu Yang, College of Information Science and Engineering, Northeastern University, Shenyang 110004, China. Tel.: +86 024-83665418; Fax: 83663446; E-mail: yangjinzhu@ise.neu.edu.cn.

0959-2989/14/\$27.50 @ 2014 - IOS Press and the authors. 
lar disease: Digital Subtraction Angiography (DSA), Trans Cranialcolor Doppler (TCD), Magnetic Resonance Angiography (MRA) and Computed Tomography Angiography (CTA) respectively, out of which MRA is the most widely used in the diagnosis of vascular disease in the Clinical practices. The precise segmentation of cerebral vascular plays an important role in diagnosis of cerebrovascular disease and therefore cerebrovascular segmentation has been a research focus recently.

The blood vessel can be segmented directly based on grayvalue [2-4], prior knowledge $[5,6]$, deformable model $[7,8]$ and graphical linking technology [9]. However, due to the fuzzy, uneven features of MRA images, vascular linear geometric feature is usually used to enhance the blood vessels before the segmentation for the extraction of brain blood vessels accurately [10,11]. Hessian matrix is one of the effective vessel enhancement methods [12-14], but it is used only ocal geometric feature of the image. As the vascular structures are enhanced, the other tubular structure like nasal soft tissue also enhanced simultaneously during this process. Thus some of the pseudo blood vessels, as well as much isolated noise points are enhanced, which certainly increase the difficulties of cerebrovascular segmentation. This proposed method is an improved Hessian multiscale enhancement filter, which combines the global grayscale information with the local geometric features. This improved filter can effectively reduce the number of the pseudo vessel structures and isolated noise points in the process of blood vessel enhancement. The proposed method in this work provides a strong foundation for the subsequent accurate segmentation of blood vessels.

\section{Method}

\subsection{Hessian multiscale enhancement filter}

Computation of Hessian matrix needs to calculate the second-order partial derivatives of the image. In general, linear scale spaces theory is applied to compute the differential operator of Hessian matrix. Under this theory, differentiation is usually defined as the convolution of raw data and derivative of a Gaussian filter. The second directional order derivative is defined as:

$$
\begin{aligned}
& \mathrm{G}(\boldsymbol{x}, \sigma)=\exp \left(-\frac{\|\boldsymbol{x}\|^{2}}{2 \sigma^{2}}\right) \\
& I_{x x}(X)=I(X) * \frac{\partial^{2} \mathrm{G}(\boldsymbol{x}, \sigma)}{\partial x^{2}}
\end{aligned}
$$

Where standard deviation $\sigma=12,3,4,5$.

The computation of eigenvalues of Hessian matrix is related with $\sigma$. Vascular enhancement process can be viewed as a process of filtering. In order to determine whether a point $X$ belongs to the tubular structure in the image, the local properties of the point should be analyzed. Eigenvalues of the Hessian matrix $\lambda_{1}, \lambda_{2}, \lambda_{3}\left(\lambda_{1}<\lambda_{2}<\lambda_{3}\right)$ and the corresponding eigenvectors, $e_{1}, e_{2}, e_{3}$ indicates the direction information of the image. Ideal tubular structure has the following characteristics:

$$
\left|\lambda_{1}\right| \approx 0 ;\left|\lambda_{1}\right| \ll\left|\lambda_{2}\right| ; \lambda_{2} \approx \lambda_{3}
$$


The symbols $\lambda_{2}$ and $\lambda_{3}$ are determined by the relations between tubular object and the background: When the objects bright and the background are dark, these two eigenvalues are negative, and they are positive for the opposite condition. Typically, blood vessels are bright and the background is dark in MRA and CTA data. The 3D vesselness function defined in traditional Hessian multiscale filtering [6]:

$$
\begin{aligned}
& v_{0}(\lambda)=\left\{( 1 - \operatorname { e x p } ( - \frac { R _ { \mathrm { A } } ^ { 2 } } { 2 \alpha ^ { 2 } } ) ) \left(\operatorname{if} \lambda_{2}>0 \text { or } \lambda_{3}>0\right.\right. \\
& \left.\left.R_{\mathrm{A}}=\left|\frac{\lambda_{2}}{\lambda_{3}}\right|, \quad R_{B}^{2}\right)\right)\left(1-\exp \left(-\frac{S^{2}}{2 \beta^{2}}\right)\right) \\
& R_{B}=\frac{\left|\lambda_{1}\right|}{\sqrt{\left|\lambda_{2} \lambda_{3}\right|}}, \quad S=\sqrt{\lambda_{1}^{2}+\lambda_{2}^{2}+\lambda_{3}^{2}} . \alpha=0.5, \beta=0.5, \quad c=20 \text { in this paper. The value }
\end{aligned}
$$

of $v_{0}(\lambda)$ is between 0 and 1 . If a pixel belongs to a vessel structure, the value of the vesselness function is closed to 1 . Otherwise, it is closed to 0 . In this way, the vascular structure can be enhanced.

The $2 \mathrm{D}$ vesselness function defined in traditional Hessian multiscale filtering:

$$
v_{0}(\lambda)=\left\{\begin{array}{cl}
0 & \text { if } \lambda_{2}>0 \\
\left(\exp \left(-\frac{R_{B}{ }^{2}}{2 \beta^{2}}\right)\right)\left(1-\exp \left(-\frac{S^{2}}{2 c^{2}}\right)\right)
\end{array}\right.
$$

Where $R_{B}=\frac{\left|\lambda_{1}\right|}{\left|\lambda_{2}\right|}, S=\sqrt{\lambda_{1}^{2}+\lambda_{2}^{2}}, \beta=0.5, c=20$.

\subsection{Improved Hessian multiscale enhancement filter}

Original Hessian multiscale enhancement filter is simply based on the local geometry feature of the image, but does not take the grayscale information of the image into account. That is the reason why some pseudo blood vessels and many isolated noise points appear in the enhancement result. Therefore, gray level factor $F$ is added in this proposed method, which is shown in the Eq. (6).

$$
F=\frac{1}{2}\left(1+\frac{2}{\pi} \arctan \left(\frac{I_{(x, y, z)}-H u}{P_{\max }} * \gamma\right)\right)
$$




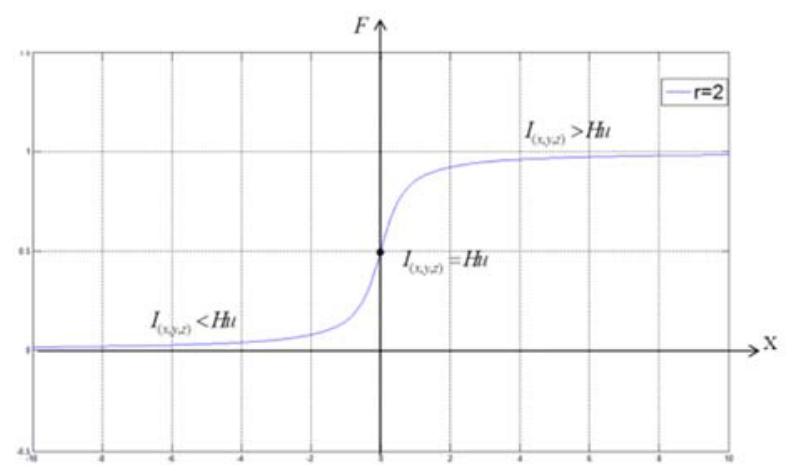

Fig. 1. The curve of $F$.

$I_{(x, y, z)}$ is the gray value of pixel point $(x, y, z) . H u$ is the global adaptive threshold of vessel obtained by adaptive iterative threshold method. $P_{\max }$ is the maximum gray value of MRA data, $\gamma$ is the smoothness factor of $F$. Where $X=\frac{I_{(x, y, z)}-H u}{P_{\max }}, \gamma=2$, the curve of $F(X)$ is depicted in Figure 1.

The parameter $H u$ in $F$ is computed by adaptive iterative threshold method. Adaptive iterative threshold method is an automatic search method of threshold. Firstly, it calculates the average image gray value and is used to segment the original image into foreground and background. Subsequently, a new threshold $\mathrm{c}$ is being evaluated by averaging integrations of these two parts. This process is repeated until the threshold value shows some change. This is represented by a mathematical expression as follows.

$$
\mathrm{T}_{i+1}=\frac{1}{2}\left[\frac{\sum_{k=0}^{T_{i}} h_{k} \cdot k}{\sum_{k=0}^{T_{i}} h_{k}}+\frac{\sum_{k=T_{i}+1}^{L-1} h_{k} \cdot k}{\sum_{k=T_{i}+1}^{L-1} h_{k}}\right]
$$

Where $L$ is the number of gray scales, and $h_{k}$ is the number of pixels with gray $k$. The iteration is continued until $T_{i}=T_{i+1} . T_{i}$ is the final segmentation threshold. $H u=T_{i}$. The result of adaptive iterative threshold method is shown in Figure 2.

The improved Hessian enhancement filtering defined in this paper is represented as shown in Eq. (8).

$$
v_{0}(\lambda)=\left\{\begin{array}{c}
0, \quad \text { if } \lambda_{2}>0 \text { or } \lambda_{3}>0 \\
\left(1-\exp \left(-\frac{R_{\mathrm{A}}{ }^{2}}{2 \alpha^{2}}\right)\right) \cdot\left(\exp \left(-\frac{R_{B}{ }^{2}}{2 \beta^{2}}\right)\right) \cdot\left(1-\exp \left(-\frac{S^{2}}{2 c^{2}}\right)\right) \cdot F
\end{array}\right.
$$




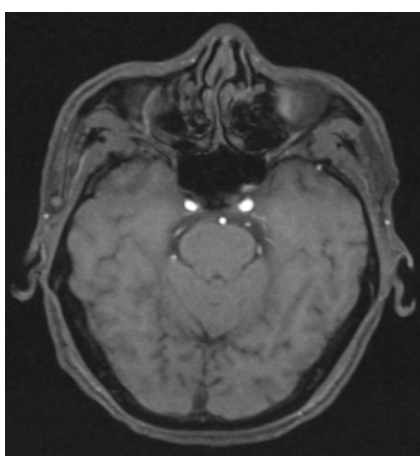

(a)

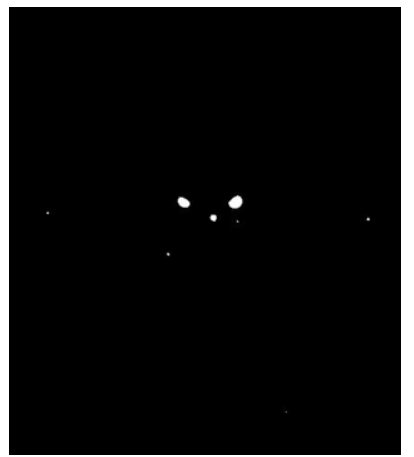

(b)

Fig. 2. The result of adaptive iterative threshold method. (a) original image and (b) the result of adaptive iterative threshold method.

$$
R_{\mathrm{A}}=\left|\frac{\lambda_{2}}{\lambda_{3}}\right|, \quad R_{B}=\frac{\left|\lambda_{1}\right|}{\sqrt{\left|\lambda_{2} \lambda_{3}\right|}}, S=\sqrt{\lambda_{1}^{2}+\lambda_{2}^{2}+\lambda_{3}^{2}}, \alpha=0.5, \beta=0.5, c=20 .
$$

Applying the operator of $F$, the pixels whose gray value are larger than $H u$ can be enhanced further, and pixels whose gray value are smaller than $H u$ will be supressed. As a result, thepseudo vessel structures and isolated noise points can be eliminated.

The 3D vesselness function defined for proposed method is represented in Eq. (9).

$$
\begin{aligned}
& v_{0}(\lambda)=\left\{\left(1-\exp \left(-\frac{R_{\mathrm{A}}^{2}}{2 \alpha^{2}}\right)\right) \cdot\left(\exp \left(-\frac{R_{B}^{2}}{2 \beta^{2}}\right)\right) \cdot\left(1-\exp \left(-\frac{S^{2}}{2 c^{2}}\right)\right) \cdot F\right. \\
& R_{\mathrm{A}}=\left|\frac{\lambda_{2}}{\lambda_{3}}\right|, \quad R_{B}=\frac{\left|\lambda_{1}\right|}{\sqrt{\left|\lambda_{2} \lambda_{3}\right|}}, S=\sqrt{\lambda_{1}^{2}+\lambda_{2}^{2}+\lambda_{3}^{2}}, \alpha=0.5, \beta=0.5, c=20
\end{aligned}
$$

The above proposed method can be applied as follow: Step 1: Convolute the image with multiscale Gaussian filter; Step 2: Compute the adaptive threshold based on Eq. (7); Step 3: Construct the gray level factor $F$ based on Eq. (6); Step 4: Compute the vesselness function based on Eq. (8);

\section{Results}

The experimental data set which consists of three image data: two brain MRA data and one lung CTA data is provided by Beijing Xuanwu hospital. This data set is used here for the improved Hessian multiscale filter in a test platform based on a PC with CPU frequency $3.4 \mathrm{GHz}$ and RAM size $4 \mathrm{~GB}$. 


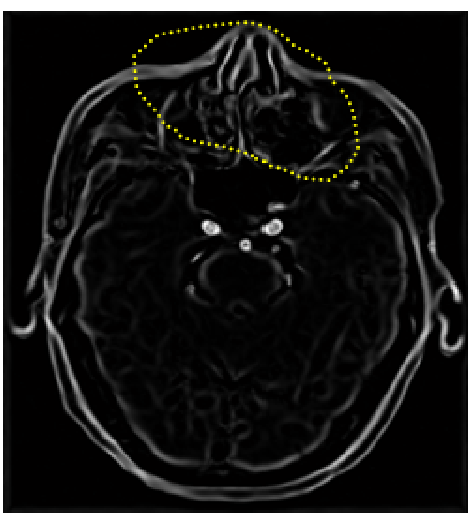

(a)

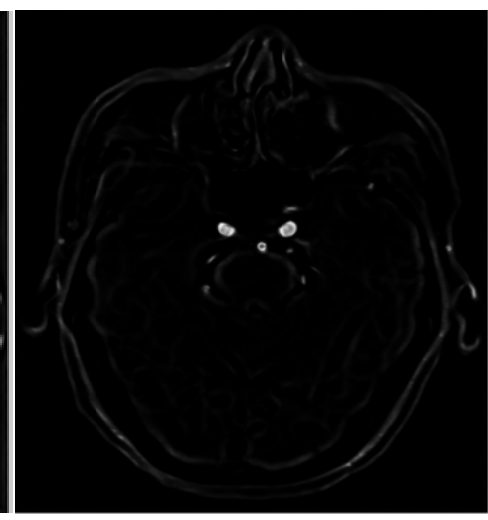

(b)

Fig. 3. Comparison of 2D improved Hessian multi-scale filtering and traditional 2D multi-scale filtering. (a) the result of one slice of data A with traditional multi-scale filtering and (b) the result of one slice of data A with improved Hessian multiscale filtering, with $\beta=0.5, c=20, \gamma=2, \sigma=1,2,3,4$.

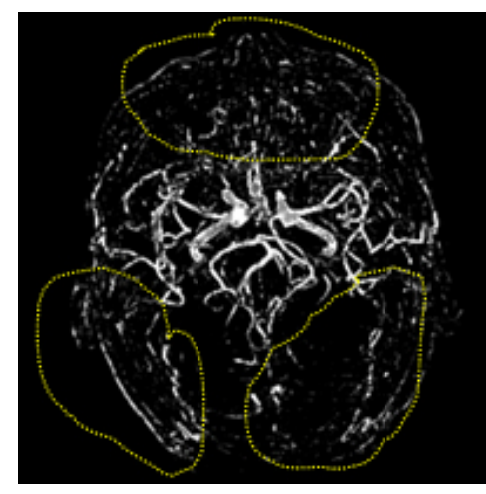

(a)

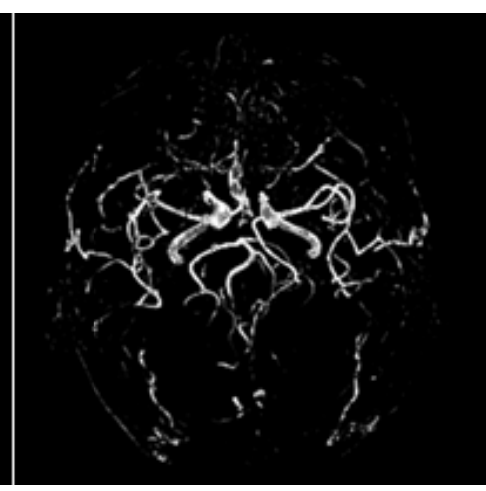

(b)

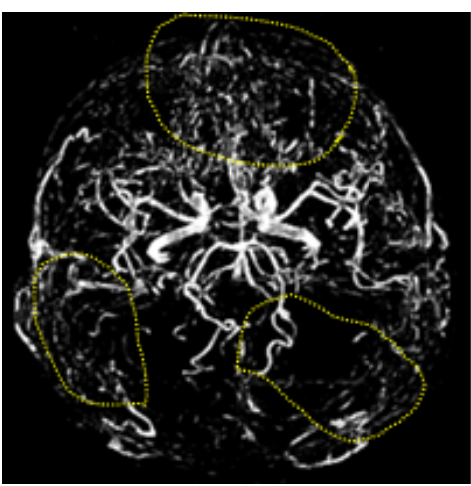

(c)

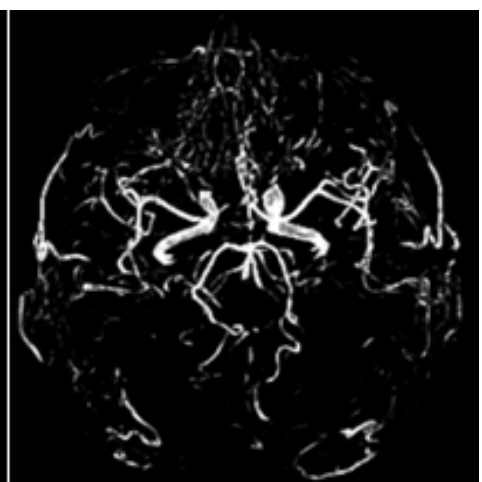

(d)

Fig. 4. Comparison of the 3D improved Hessian multi-scale filtering and traditional 3D multi-scale filtering. (a) the result of data A with traditional multi-scale filtering, (b) the result of data A with improved Hessian multi-scale filtering, (c) the result of data $\mathrm{B}$ with traditional multi-scale filtering and (d) the result of data $\mathrm{B}$ with improved Hessian multi-scale filtering, with $\alpha=0.5, \beta=0.5, c=20, \gamma=2, \sigma=1,2,3,4,5$. 


\subsection{The enhancement results of brain MRA}

The two sets of brain MRA data (data A and data B) are enhanced by the improved method. The resolution of data $A$ is $352 \times 448 \times 92$ and data $B$ is $352 \times 448 \times 102$. Figure 3 shows the comparison result of 2D improved Hessian multiscale filter and traditional 2D Hessian multiscale filter, whereas the original image is shown in Figure 2. It is observed from the results, that the proposed method enhances vascular region, and, reduces the pseudo vessels simultaneously such as the nasal soft tissue and the brain border.

Figure 4 shows the comparison result of the 3D improved Hessian multiscale filter and traditional 3D multiscale filter. The yellow marked region shows that the proposed method can effectively reduce pseudo vessels and isolated noise points that produced by enhancement.

\subsection{The enhancement results of lung $C T$}

One set of Lung CTA data provided by Xuanwu Hospital is used to further assess the improved Hessian multiscale filter. The resolution of this data is $512 * 512 * 345$. Figure 5 is the comparison result of 2D improved Hessian multiscale filter and traditional 2D Hessian multiscale filter. Figure 6 shows the comparison result of the 3D image. From the experimental results, it is derived that the method can effectively reduce the enhancement of the border and isolated noise points that produced by Hessian filter.

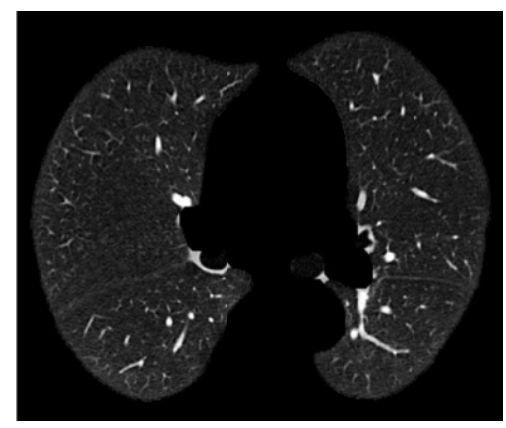

(a)

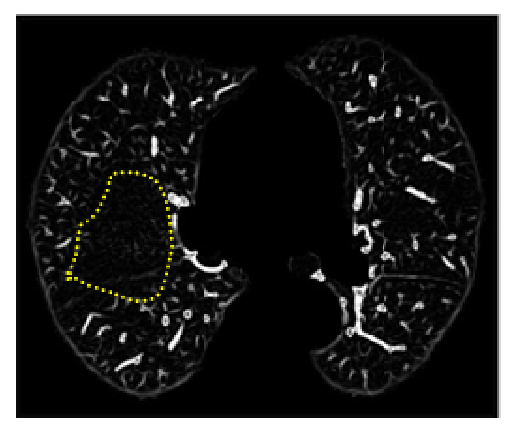

(c)

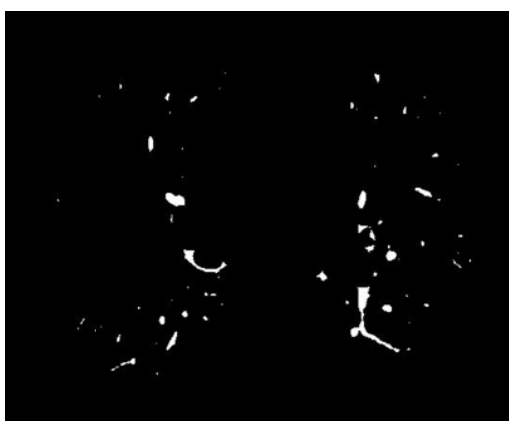

(b)

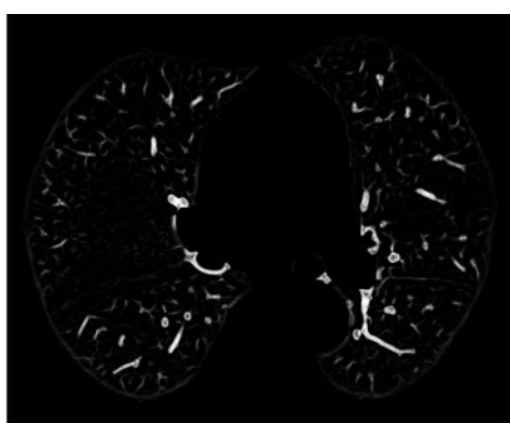

(d)

Fig. 5. Comparison of 2D improved Hessian multi-scale filtering and traditional 2D multi-scale filtering. (a) original image, the result of adaptive iterative threshold method, (c) the result of one slice of CT data with traditional multi-scale filtering and (d) the result of one slice of CT data with improved, with $\beta=0.5, c=20, \gamma=2, \sigma=1,2,3,4$. 


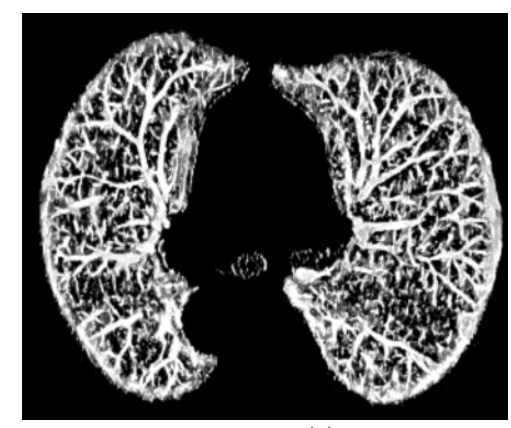

(a)

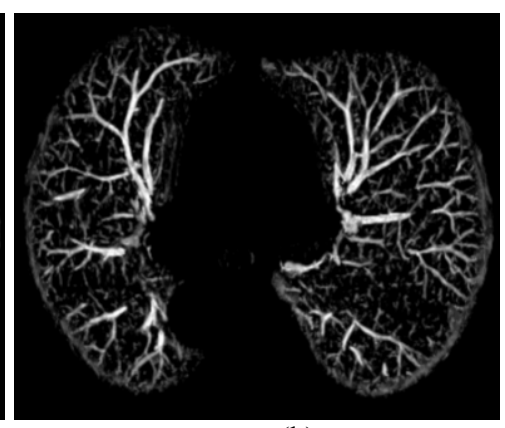

(b)

Fig. 6. Comparison of the 3D improved Hessian multi-scale filtering and traditional 3D multi-scale filtering. (a) the result of 21 slices of CT data with traditional multi-scale filtering and (b) the result of 21 slices of CT data with improved Hessian multi-scale filtering, with $\alpha=0.5, \beta=0.5, c=20, \gamma=2, \sigma=1,2,3,4,5$.

Experimental results are being evaluated by clinical doctors of Xuanwu Hospital and the Doctors opine that the proposed method can effectively reduce the pseudo vessel structures and isolated noise points, and gives a better than the traditional Hessian multi-scale filtering.

\subsection{The segmentation result of adaptive threshold method}

The proposed method in this paper can effectively reduce the pseudo vascular structures and isolated noise points caused by the multiscale filter. But due to some noise points exist in the enhanced image, an adaptive threshold method is adopted in the process of vessel segmentation. To further reduce the isolated noise points, the connected domain search algorithm in three-dimensional space [15-17] is applied. The vessel extraction results of the brain and the lung are shown respectively in Figure 7.

\section{Conclusion}

In this paper, an improved Hessian multiscale filter is proposed by combining the global grayscale information and the local geometric feature. The experimental results show that the proposed method can effectively reduce the pseudo vessel structures and isolated noise points. As a result, the segmentation is more precise and meaningful for clinical diagnosis.

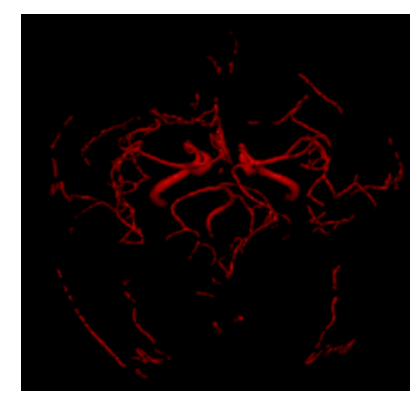

(a)

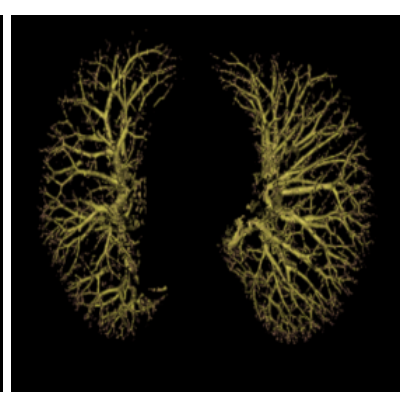

(b)

Fig. 7. Results of cerebrovascular extraction and results of lung vessel extraction. (a) the segmentation result of brain vessel and (b) the segmentation result of lung vessel with 61 slices of CT data. 


\section{Acknowledgement}

We are thankful to Xuanwu Hospital to for providing experimental data. This work is supported by the Fundamental Research Funds for the Central Universities under grant N120518001, Chinese National Natural Science Foundation under grant No. 61001047 and No. 81271556, Liaoning Natural Science Foundation under grant 2013020021, LJQ2013026.This work is also supported by the National Key Technology Research and Development Program of the Ministry of Science and Technology of China under grant No. 2014BAI17B02.

\section{References}

[1] The National Cardiovascular Disease Center, The China cardiovascular disease report 2012, China, 2012.

[2] D.L. Wilson and J.A. Noble, An adaptive segmentation algorithm for time-of-flight MRA data, IEEE Trans. Med. Imaging 18 (1999), 938-945.

[3] M.S. Hassouna, A.A. Farag and S. Hushek, Cerebrovascular segmentation from TOF using stochastic models, Med. Image Anal. 10 (2006), 2-18.

[4] R. Gan, W.C. Wong and A.C. Chung, Statistical cerebrovascular segmentation in three-dimensional rotational angiography based on maximum intensity projections, Med. Phys. 32 (2005), 3017-3028.

[5] S.C. Faber, A. Hoffmann and C. Ruedig, MRI-induced stimulation of peripheral nerves: Dependency of stimulation threshold on patient positioning, Magn. Reson. Imaging 21 (2003), 715-724.

[6] N. Passat, C. Ronse and J. Baruthio, Watershed and multimodal data for brain vessel segmentation: Application to the superior sagittal sinus, Image Vis. Comput. 25 (2007), 512-521.

[7] C. Li, R. Huang, Z. Ding, C. Gatenby, D. N. Metaxas and J. C. Gore, A level set method for image segmentation in the presence of intensity in homogeneities with application to MRI, IEEE Trans. Image Processing 20 (2011), 2007-2016.

[8] C. Li, C. Xu, C. Gui and M.D. Fox, Distance regularized level set evolution and its application to image segmentation, IEEE Trans. Image Processing 19 (2010), 3243-3254.

[9] Z.J. Cheng, Automated delineation of calcified vessels in mammography by tracking with uncertainty and graphical linking techniques, IEEE Transactions of Medical Imaging 31 (2012), 2143-2155.

[10] S.D. Olabarriaga, M. Breeuwer and W.J. Niessen, Evaluation of Hessian-based filters to enhance the axis of coronary arteries in CT images, International Congress Series, Elsevier 1256 (2003), 1191-1196.

[11] R. Van and M. Eva, Supervised enhancement filters: Application to fissure detection in chest CT scans, IEEE Transactions of Medical Imaging 27 (2008), 1-10.

[12] M. Rashindra and W. Niessen, Multiscale vessel enhancing diffusion in CT angiography noise filtering, Information Processing in Medical Imaging, Springer Berlin Heidelberg (2005).

[13] R.F. Frangi, W.J. Niessen and K.L. Vincken, Multiscale vessel enhancement filtering, Medical Image Computing and Computer-Assisted Intervention 1496 (1988), 130-137.

[14] P.T.H Truc, M.A.U Khan and Y.K. Lee, Vessel enhancement filter using direction filter bank, Computer Vision and Image Understanding 113 (2009), 101-112.

[15] J.Z. Yang, T.G. Paloma and H.C. Peng, A distance-field based automatic neuron tracing method, BMC Bioinformatics, 14 (2013), 93-104.

[16] D. Babin, A. Pižurica and V.J. De, Brain blood vessel segmentation using line-shaped profiles, Physics in Medicine and Biology 58 (2013), 8041-8052.

[17] X. Wu, V. Luboz and K. Krissian, Segmentation and reconstruction of vascular structures for 3D real-time simulation, Medical Image Analysis 15 (2011), 22-34. 